“( 2015 IEEE. Personal use of this material is permitted. Permission from IEEE must be obtained for all other uses, in any current or future media, including reprinting/republishing this material for advertising or promotional purposes, creating new collective works, for resale or redistribution to servers or lists, or reuse of any copyrighted component of this work in other works." 


\title{
Survey of Error Concealment Techniques: Research Directions and Open Issues
}

\author{
Muhammad Usman ${ }^{\Psi} \quad$ Xiangjian $\mathrm{He}^{\Psi} \quad \operatorname{Min~Xu}^{\Psi} \quad$ Kin Man Lam \\ $\Psi$ School of Computing and Communication, University of Technology, Sydney, Australia \\ $\dagger$ Department of Electronic and Information Engineering, The Hong Kong Polytechnic University, Kowloon, Hong Kong

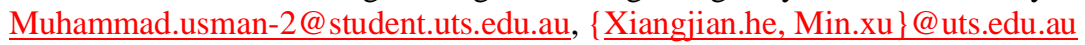 \\ Kmlam@polyu.edu.hk
}

\begin{abstract}
Error Concealment (EC) techniques use either spatial, temporal or a combination of both types of information to recover the data lost in transmitted video. In this paper, existing EC techniques are reviewed, which are divided into three categories, namely Intra-frame EC, Inter-frame EC, and Hybrid EC techniques. We first focus on the EC techniques developed for the H.264/AVC standard. The advantages and disadvantages of these EC techniques are summarized with respect to the features in H.264. Then, the EC algorithms are also analyzed. These EC algorithms have been recently adopted in the newly introduced H.265/HEVC standard. A performance comparison between the classic EC techniques developed for $\mathrm{H.264}$ and H.265 is performed in terms of the average PSNR. Lastly, open issues in the EC domain are addressed for future research consideration.
\end{abstract}

Keywords-Error concealment; Spatial; Temporal; H.264/AVC; H.265/HEVC

\section{INTRODUCTION}

Nowadays, compressed data communication is very common because of the limitations on available bandwidth. Some applications, e.g. video conferencing, require a large bandwidth to transmit high-quality videos. To cope with the problem of available limited bandwidth, videos are usually compressed before transmission. The compressed format of videos, which is called a bit stream, is very sensitive to single or multiple bit errors. Such errors are very common in wireless communication channels and have become a major challenge to deal with. In IP-based packet networks, delays are usual, which disturbs synchronization at the decoder side, leading to unsettled visual quality in both subjective and objective ways. Video coding standards, such as MPEG-X and H.26X, usually use prediction and hybrid coding methods to encode a video, and generate a compressed bit stream. If errors occur in some frames during transmission, there will be a quality loss in all of the subsequent frames; this requires prediction to be performed for the damaged frames [1]. Motion compensation in the temporal domain plays an important role in error propagation in the future frames, because these frames obtain the motioncompensation information from their previous or reference frames.

To deal with errors, which cause packet loss in video transmission over wireless communication channels, the
Forward Error Correction (FEC) and Automatic Retransmission Request (ARQ) techniques were used initially [2]. These techniques require the availability of high bandwidth and are vulnerable to residual losses. The ARQ technique demands a feedback channel between an encoder and a decoder. Both FEC and ARQ techniques are time-consuming and are not suitable for real-time communication because of their operating nature [3]. The error resilient schemes based on Distributed Source Coding (DSC) play an important role against propagation errors in predictive video coding. Channelaware video coding based on DSC is proposed to eliminate propagation errors [4]. Such techniques are used useful against propagation errors but cannot deal with hardware errors, e.g. buffer overloading, hardware and power failures. Intensive research has been undertaken to develop EC techniques that are compatible with real-time communication and do not demand high bandwidth. EC techniques work as a post-process at the decoder side, and utilize spatial and/or temporal information from video sequences. This paper focuses on recent developments in EC techniques.

The rest of this paper is organized as follows. Section II categorizes the classic EC schemes into three categories based on the information being utilized. Section III discusses the latest EC algorithms developed in terms of the new features in H.265. Section IV concludes the paper and discusses future research directions.

\section{CLASSIC ERROR CONCEALMENT TECHNIQUES}

This section summarizes some classic EC techniques. These techniques are then discussed and compared in terms of their features and applicable environments.

\section{A. Intra-Frame Error Concealment Techniques}

Intra-frame EC is also known as Spatial EC. Spatial EC techniques are pixel-based, i.e. the information from the surrounding group of pixels in the same frame is used to estimate those lost/damaged pixels. Such EC techniques use either texture features or edge/object-shape information. A special case occurs when most of the surrounding pixels are also damaged or lost, and cannot provide useful information for prediction. In such a case, previously processed frame(s) 
can be used to estimate the lost information. A summary of Intra-frame EC techniques, along with their advantages and disadvantages, can be found in Table I.

The textures-based techniques, which usually use statistical methods, can be classified into statistical, structural and modelbased techniques. In the statistical approach, the statistical results of selected features are merged and processed to predict the features of a missing region. In the structural approach, textures are classified as visually strong or weak textures. This categorization of structure helps in estimating the texture of foreground and background regions. In texture modeling, the texture of an image or a texture image is modeled as a linear combination of basis functions or a probability model. The coefficients of such models help in categorizing different textures [5]. A texture model based on geometric interpolation is used to recover damaged pixels by using image textures from neighboring pixels in [6]. This technique produces defined number of computations. However, it works well with larger block sizes and requires feedback interaction with the encoder. Spatial EC based on convex optimization is used to reconstruct missing Macro Blocks (MBs) by using a weighted set of templates from neighborhood blocks in [7]. This technique works well in both the spatial and temporal domains. In addition, the computational complexity of this technique can be minimized by using several approximations. The Probability Density Function (PDF) from Kernel Density Estimation (KDE) and the minimum Mean Error Square Estimator (MESE) are used to reconstruct the lost portion of an Intraframe with vector formalism in [8]. This approach requires more computational complexity to improve reconstruction quality. The approaches presented in [6-8] produce better reconstruction quality but are not suitable for real-time processing.

There are certain points in images where the brightness changes rapidly. Such points are grouped into curved lines, known as edges. Mathematical techniques to detect edges are called edge or contour or boundary detection techniques [9]. Directional edge analysis is used to locate edges of missing areas, and the weighted average of the two corresponding edges is used to conceal missing areas in [10]. This approach produces a defined number of computations, and is suitable for images having significant edges with details. The Canny edge detector is used to detect relevant edges in order to conceal digital dropout errors for the content of digital video tapes in [11]. This technique can restore complex edges and non-linear features with controlled computational complexity. However, it relies on Pathological Motion (PM). Relevant edges are found by using the Hough Transform, and fine concealment is achieved through repeated interpolations in [12]. This approach can reconstruct complex textures, but is computationally complex and requires accelerated hardware. The approaches presented in [10-12] produce a better reconstruction performance with simple operations and are suitable for the random loss of data.

Disparity matching is performed between two images on a pixel-by-pixel basis to recover lost MBs in [13]. This technique works well for the random loss of MBs. Binary decision tree is used to select suitable EC algorithm along with a reference frame to conceal missing MBs in [14]. This technique is adaptive in nature, but causes a high computational complexity. The approaches presented in [13-14] produce better reconstruction quality, but are not suitable for real-time processing.

TABLE I. Intra-frame error concealment technique

\begin{tabular}{|c|c|c|c|}
\hline $\begin{array}{c}\text { Approach } \\
\text { es }\end{array}$ & $\begin{array}{c}\text { Technique } \\
\text { s }\end{array}$ & Advantages & Disadvantages \\
\hline \multirow{3}{*}{$\begin{array}{l}\text { Statistical } \\
\text { Approache } \\
\mathrm{s}\end{array}$} & $\begin{array}{l}\text { Textures of } \\
\text { neighborin } \\
\text { g pixels [6] }\end{array}$ & \multirow{3}{*}{$\begin{array}{c}\text { Simple } \\
\text { interpolation } \\
\text { s, Controlled } \\
\text { computationa } \\
\text { l complexity, } \\
\text { Suitable for } \\
\text { single } \\
\text { images }\end{array}$} & \multirow{3}{*}{$\begin{array}{l}\text { Suitable for large block sizes, } \\
\text { Iterative processing, } \\
\text { Unsuitable with Consecutive } \\
\text { errors, Sometimes unsuitable } \\
\text { for real-time video processing }\end{array}$} \\
\hline & $\begin{array}{l}\text { Weighted } \\
\text { set of } \\
\text { templates } \\
\text { of } \\
\text { neighborin } \\
\text { g pixels [7] }\end{array}$ & & \\
\hline & $\begin{array}{l}\text { Vector } \\
\text { samples } \\
\text { from } \\
\text { neighborin } \\
\text { g pixels [8] }\end{array}$ & & \\
\hline \multirow{3}{*}{$\begin{array}{l}\text { Boundary } \\
\text { Detection } \\
\text { Approache } \\
\text { s }\end{array}$} & $\begin{array}{l}\text { Weighted } \\
\text { average of } \\
\text { edges [10] }\end{array}$ & \multirow{3}{*}{$\begin{array}{c}\text { Mathematica } \\
\text { lly simple } \\
\text { operations, } \\
\text { Controlled } \\
\text { computationa } \\
\text { l complexity, } \\
\text { Reconstruct } \\
\text { complex } \\
\text { edges and } \\
\text { non-linear } \\
\text { features and } \\
\text { textures } \\
\end{array}$} & \multirow{3}{*}{$\begin{array}{l}\text { Dependent on significant } \\
\text { edges details, Depends upon } \\
\text { PM, Iterative processing, } \\
\text { Unsuitable for consecutive } \\
\text { errors, Sometimes unsuitable } \\
\text { for real-time video processing }\end{array}$} \\
\hline & $\begin{array}{c}\text { Canny } \\
\text { edge } \\
\text { detector } \\
{[11]} \\
\end{array}$ & & \\
\hline & $\begin{array}{l}\text { Hough } \\
\text { Transform } \\
{[12]}\end{array}$ & & \\
\hline \multirow{2}{*}{$\begin{array}{l}\text { Past Frame } \\
\text { Based } \\
\text { Approache } \\
\text { s }\end{array}$} & $\begin{array}{l}\text { Pixel } \\
\text { matching } \\
{[13]}\end{array}$ & \multirow{2}{*}{$\begin{array}{l}\text { Suitable for } \\
\text { random } \\
\text { errors, } \\
\text { Adaptive in } \\
\text { nature }\end{array}$} & \multirow{2}{*}{$\begin{array}{c}\text { Partially real-time, } \\
\text { Computationally complex, } \\
\text { Unsuitable for real-time video } \\
\text { processing }\end{array}$} \\
\hline & $\begin{array}{c}\text { Multiple } \\
\text { EC } \\
\text { algorithms } \\
{[14]}\end{array}$ & & \\
\hline
\end{tabular}

\section{B. Inter-Frame Error Concealment Techniques}

Inter-Frame EC is also known as Temporal EC. Temporal EC techniques are usually used to recover lost Motion Vectors (MVs), which are used to recover damaged MBs by considering the MVs of corresponding MBs in a reference frame. However, these techniques can also use algorithms from other domains of image processing to refine or filter out estimated MVs. Temporal EC techniques perform better than spatial EC techniques when the motion is continuous in the consecutive frames. Such EC techniques are used in both 2-D and 3-D video domains. Table II summaries current Interframe EC techniques along with their advantages and disadvantages.

A 2-D image is an image, captured through a camera with the height and width information. Videos made up of a combination of such images are called 2-D videos [15]. In [16], object detection, the boundary matching score, and MVs from a reference frame are used to recover the lost MBs of variable sized in a current frame. This approach performs better on 
frames having multiple objects. An iterative Dynamic Programming (DP)-based approach is used to estimate the lost MVs of corrupted MBs in [17]. This technique works well with both Intra and Inter-frames. In [18], the MVs from 20 nearby sub-blocks are used to restore the MVs of a lost MB within the same frame. This technique can be applied to real-time applications, but is not suitable for videos having slow motion and a consecutive loss of MBs. The MVs of a lost MB are derived from a reference MB by using any recovery algorithm, and are then refined by auto-regressive modeling in [19]. This approach works well with a consecutive loss of MBs. The MVs of missing MBs are predicted by using a Kalman filter in combination with modified bilinear motion field interpolation (MFI) in [20]. This approach works well with heavily corrupted videos, but is suitable only for videos having linear motion. Slow and fast-moving regions in a current frame are identified to estimate the missing region in [21]. This method is suitable for whole-frame loss, but unsuitable for consecutive loss. The approaches presented in [16-21] produce a better reconstruction quality. The approaches presented in [16-17, 1921] are computationally expensive and unsuitable for real-time processing.

TABLE II. Inter-frame error concealment techniques

\begin{tabular}{|c|c|c|c|}
\hline $\begin{array}{l}\text { Approach } \\
\text { es }\end{array}$ & Techniques & $\begin{array}{l}\text { Advanta } \\
\text { ges }\end{array}$ & $\begin{array}{l}\text { Disadvanta } \\
\text { ges }\end{array}$ \\
\hline \multirow{6}{*}{$\begin{array}{l}\text { 2-D Video- } \\
\text { Based } \\
\text { Approaches }\end{array}$} & $\begin{array}{c}\text { Object detection } \\
\text { using MVs from } \\
\text { reference frame } \\
{[16]}\end{array}$ & \multirow{6}{*}{$\begin{array}{c}\text { Suitable for } \\
\text { multiple- } \\
\text { object } \\
\text { detection and } \\
\text { both Intra and } \\
\text { Inter-frames, } \\
\text { Suitable with } \\
\text { consecutive } \\
\text { errors and } \\
\text { with whole } \\
\text { frame loss }\end{array}$} & \multirow{6}{*}{$\begin{array}{l}\text { Computationally } \\
\text { expensive, } \\
\text { Unsuitable for } \\
\text { real-time video } \\
\text { processing and } \\
\text { with non-linear } \\
\text { motion }\end{array}$} \\
\hline & Iterative DP [17] & & \\
\hline & $\begin{array}{l}\text { Auto-regressive } \\
\text { modeling with } \\
\text { MVs from } \\
\text { reference frame } \\
{[18]}\end{array}$ & & \\
\hline & Kalman filter [19] & & \\
\hline & $\begin{array}{c}\text { Region } \\
\text { distribution based } \\
\text { upon motion [20] }\end{array}$ & & \\
\hline & $\begin{array}{c}\text { MVs of } \\
\text { neighboring MBs } \\
{[21]}\end{array}$ & & \\
\hline \multirow{4}{*}{$\begin{array}{l}\text { 3-D Video- } \\
\text { Based } \\
\text { Approaches }\end{array}$} & $\begin{array}{c}\text { Depth image } \\
\text { information [23] }\end{array}$ & \multirow{4}{*}{$\begin{array}{l}\text { Suitable for } \\
\text { whole-frame } \\
\text { loss, No } \\
\text { dependency } \\
\text { on reference } \\
\text { frame }\end{array}$} & \multirow{4}{*}{$\begin{array}{c}\text { Unsuitable with } \\
\text { 2-D videos and } \\
\text { for real-time } \\
\text { video } \\
\text { processing, } \\
\text { Dependent on } \\
\text { depth } \\
\text { information, } \\
\text { Computationally } \\
\text { complex }\end{array}$} \\
\hline & $\begin{array}{l}\text { MVs from past } \\
\text { Intra-frames [24] }\end{array}$ & & \\
\hline & $\begin{array}{c}\text { MVs variance and } \\
\text { textures from } \\
\text { neighboring MBs } \\
{[25]}\end{array}$ & & \\
\hline & $\begin{array}{l}\text { Intra and Inter } \\
\text { MVE [26] }\end{array}$ & & \\
\hline
\end{tabular}

A 3-D image includes the perception of depth, as compared with a 2-D image. Videos made up of such images are called 3$\mathrm{D}$ videos. In the past, dual cameras were used to produce such videos. However, nowadays this is usually done by using software technology, which transforms 2-D videos to 3-D videos [15]. An analysis of 3-D video compression, transmission and quality evaluation is summarized in [22]. The depth-image information is used to estimate the lost MVs in 3-
D videos [23]. This approach works well with whole-frame loss and real-time streaming. Another similar approach considers past Intra-frames as a reference in 3-D videos to recover the lost MVs in a current Intra-frame in [24]. This method can reduce the computational complexity. In [25], the MV variances and texture histograms from nearby MBs are used to estimate the similarity between the lost and nearby MBs in 3-D videos. This technique is less computationally complex. In [26], the lost frames of a 3-D video are recovered by using Inter and Intra-MV Extrapolation (MVE), ignoring the depth information in multimedia wireless sensor networks. The approaches presented in [23-26] produce a better reconstruction quality, but are only suitable for 3-D/multi-view video transmission.

\section{Hybrid Error Concealment Techniques}

The EC techniques proposed in the Intra and Inter domains have their own advantages and disadvantages. Sometimes, it is better to combine established EC techniques from the Intra and Inter domains or to combine techniques from other domains of signal and image processing to perform EC. Table III summaries the Hybrid EC techniques along with their advantages and disadvantages.

TABLE III. Hybrid error concealment techniques

\begin{tabular}{|c|c|c|c|}
\hline Approaches & Techniques & Advantages & Disadvantages \\
\hline $\begin{array}{l}\text { Spatio- } \\
\text { Temporal- } \\
\text { Based } \\
\text { Approaches }\end{array}$ & $\begin{array}{c}\text { Joint spatio- } \\
\text { temporal } \\
\text { technique with } \\
\text { MDC [28] } \\
\text { Joint spatio- } \\
\text { temporal } \\
\text { technique } \\
\text { without MDC } \\
{[29]}\end{array}$ & $\begin{array}{c}\text { Better } \\
\text { reconstruction } \\
\text { quality }\end{array}$ & $\begin{array}{c}\text { Computationally } \\
\text { complex, } \\
\text { Unsuitable for } \\
\text { consecutive loss of } \\
\text { data }\end{array}$ \\
\hline $\begin{array}{l}\text { Random } \\
\text { Approaches }\end{array}$ & $\begin{array}{c}\text { Gaussian } \\
\text { process with } \\
\text { signal } \\
\text { extrapolation } \\
\text { [30] } \\
\text { MDC with } \\
\text { SPIHT [31] } \\
\text { Video } \\
\text { resolution with } \\
\text { ROI [32] }\end{array}$ & $\begin{array}{c}\text { Suitable for } \\
\text { sensitive edges } \\
\text { and consecutive } \\
\text { loss of data, } \\
\text { Mathematically } \\
\text { simple, Less } \\
\text { computational }\end{array}$ & $\begin{array}{l}\text { Requires training, } \\
\text { Unsuitable for } \\
\text { real-time video } \\
\text { processing and } \\
\text { with random } \\
\text { errors, Demand } \\
\text { high bandwidth }\end{array}$ \\
\hline
\end{tabular}

In [27], an analysis of established EC techniques for H.264 encoded videos is presented to consider possible further improvements. Joint spatio-temporal EC combined with Multiple Descriptive Coding (MDC) is used to estimate lost information in [28]. A similar approach in [29] combines temporal and spatial EC techniques without using MDC to estimate lost information. The techniques proposed in [28-29] perform well, producing a better reconstruction quality with 
less computational overhead, but they do not support the consecutive loss of data.

In [30], adaptive non-stationary kernels in Gaussian processes are combined with signal extrapolation to restore corrupted data in images and videos. This approach reconstructs sensitive edges well but requires kernel training which makes it unsuitable for real-time applications. The MDC is used in combination with Set Partitioning in Hierarchical Trees (SPIHT) to perform EC on corrupted videos in [31]. High and low-resolution versions of the same video are streamed together to perform EC for Regions of Interest (ROI) in [32]. The techniques presented in [30-32] support consecutive loss of data with a high bandwidth demand.

\section{ERROR CONCEALMENT SCHEMES FOR H.265/HEVC}

There are a number of new features introduced in H.265/HEVC, such as the quad-tree structure, prediction and transform units, increased prediction directions, spatiotemporal-based MV prediction, and extra filters along with the de-blocking filter. With the help of these new features, H.265 is said to require half of the bit rate that H.264 needs, with the same visual quality. This promised bit rate makes H.265 an eye-catching development for wired and wireless transmission networks. However, the compressed bit stream generated by H.265 is very sensitive to single and multiple bit errors. This section summarizes those EC techniques based on the different features in H.265. Details of the video sequences and the features used are summarized in Table IV.

TABLE IV. Error concealment algorithms based on H.265 features

\begin{tabular}{|c|c|c|c|c|}
\hline Approaches & $\begin{array}{c}\text { Video } \\
\text { Sequence (s) }\end{array}$ & Resolution & $\begin{array}{c}\text { Test } \\
\text { Model }\end{array}$ & $\begin{array}{c}\text { Features } \\
\text { Used }\end{array}$ \\
\hline $\begin{array}{c}\text { Data Hiding } \\
\text { EC (DHEC) } \\
{[33]}\end{array}$ & $\begin{array}{c}\text { Traffic, } \\
\text { Kimono, } \\
\text { KristenAndS } \\
\text { ara, } \\
\text { RaceHorses }\end{array}$ & $\begin{array}{c}2560 \times 1600 \\
1920 \times 1080 \\
1280 \times 720 \\
832 \times 480\end{array}$ & $\begin{array}{c}\mathrm{HM} \\
9.1\end{array}$ & $\begin{array}{c}\text { Quad Tree } \\
\text { Partition, } \\
\text { Coding Units, } \\
\text { Prediction } \\
\text { Units, Parallel } \\
\text { Structures }\end{array}$ \\
\hline $\begin{array}{c}\text { Motion- } \\
\text { Compensate } \\
\text { d EC } \\
\text { (MCEC) } \\
{[34]}\end{array}$ & Soccer, Drill & $\begin{array}{l}720 \times 480, \\
832 \times 480\end{array}$ & $\begin{array}{l}\mathrm{HM} \\
11.0\end{array}$ & $\begin{array}{c}\text { Coding Units, } \\
\text { Prediction } \\
\text { Units }\end{array}$ \\
\hline $\begin{array}{c}\text { Variable } \\
\text { Block Size } \\
\text { based EC } \\
\text { (VBSEC) } \\
{[35]}\end{array}$ & $\begin{array}{l}\text { BasketballDr } \\
\text { ill, Vidyo4, } \\
\text { KristenAndS } \\
\text { ara, Café }\end{array}$ & $\begin{array}{c}832 \times 480 \\
1280 \times 720 \\
1280 \times 720 \\
1920 \times 1080\end{array}$ & $\begin{array}{c}\mathrm{HM} \\
6.0\end{array}$ & $\begin{array}{c}\text { Coding Units, } \\
\text { Prediction } \\
\text { Units }\end{array}$ \\
\hline $\begin{array}{c}\text { Partition } \\
\text { Decision } \\
\text { based EC } \\
\text { (PCEC) [37] }\end{array}$ & BQMall & $832 \times 480$ & $\begin{array}{l}\mathrm{HM} \\
5.0\end{array}$ & $\begin{array}{c}\text { Coding Units, } \\
\text { Prediction } \\
\text { Units }\end{array}$ \\
\hline
\end{tabular}

The performance comparison of those algorithms implemented in H.264 and H.265, in terms of average PSNR and Packet Loss Ratio (PLR), is tabulated in Table V. It is very clear from Table IV that these proposed techniques cannot be directly compared with each other. In order to compare the performances of different approaches, two criteria must be met: the same input video sequences and the same reference software/test model. Furthermore, computational complexity should be considered, but none the algorithms were discussed in this context. Moreover, different video sequences and different versions of the test models have been used. As shown in Table V, better PSNRs are achieved when those techniques for H.264 are re-implemented by combining them with the improved features of H.265.

TABLE V. Performance comparison based on average PSNR

\begin{tabular}{|c|c|c|c|c|}
\hline $\begin{array}{l}\text { Approach } \\
\text { es }\end{array}$ & Algorithms & $\begin{array}{c}\text { Video } \\
\text { Sequence(s) }\end{array}$ & $\begin{array}{c}\text { Average } \\
\text { PSNR } \\
\text { using } \\
\text { H.265 } \\
\end{array}$ & $\begin{array}{c}\text { Average } \\
\text { PSNR } \\
\text { using } \\
\text { H.264 } \\
\end{array}$ \\
\hline $\begin{array}{c}\text { Data } \\
\text { Hiding EC } \\
\text { (DHEC) } \\
\text { [33] }\end{array}$ & $\begin{array}{l}\text { Outer } \\
\text { Boundary } \\
\text { Matching } \\
\text { Algorithm } \\
\text { (OBMA) }\end{array}$ & $\begin{array}{c}\text { Traffic, } \\
\text { Kimono, } \\
\text { KristenAndSa } \\
\text { ra, } \\
\text { RaceHorses } \\
\text { with } 1 \% \text { PLR } \\
\end{array}$ & $\begin{array}{l}33.20 \\
34.18, \\
35.32 \\
28.79\end{array}$ & $\begin{array}{l}29.81 \\
32.58 \\
34.60 \\
26.41\end{array}$ \\
\hline $\begin{array}{c}\text { Motion } \\
\text { Compensat } \\
\text { ed EC } \\
(\mathrm{MCEC}) \\
{[34]} \\
\end{array}$ & $\begin{array}{c}\text { Motion } \\
\text { Compensat } \\
\text { ed Error } \\
\text { Concealme } \\
\text { nt (MCEC) }\end{array}$ & $\begin{array}{l}\text { Soccer, Drill } \\
\text { with } 1 \% \text { PLR }\end{array}$ & $\begin{array}{l}28.84 \\
31.17\end{array}$ & $\begin{array}{l}28.56 \\
30.86\end{array}$ \\
\hline $\begin{array}{c}\text { Variable } \\
\text { Block Size } \\
\text { based EC } \\
\text { (VBSEC) } \\
{[35]} \\
\end{array}$ & $\begin{array}{c}\text { Motion } \\
\text { Vector } \\
\text { Extrapolati } \\
\text { on (MVE) }\end{array}$ & $\begin{array}{c}\text { BasketballDri } \\
\text { ll, Vidyo4, } \\
\text { KristenAndSa } \\
\text { ra, Café with } \\
10 \% \text { PLR } \\
\end{array}$ & $\begin{array}{l}30.76, \\
36.46, \\
38.11 \\
37.92\end{array}$ & $\begin{array}{l}30.08, \\
36.23 \\
37.91 \\
37.93\end{array}$ \\
\hline $\begin{array}{c}\text { Partition } \\
\text { Decision } \\
\text { based EC } \\
(\text { PCEC) } \\
{[37]}\end{array}$ & $\begin{array}{c}\text { Motion } \\
\text { Vector } \\
\text { Extrapolati } \\
\text { on (MVE) }\end{array}$ & $\begin{array}{l}\text { BQMall with } \\
\text { only } 1 \text { P- } \\
\text { frame drop }\end{array}$ & 25.7232 & 23.701 \\
\hline
\end{tabular}

\section{A. Video Error Concealment Based on Data Hiding}

In [33], Data-Hiding-based Error Concealment (DHEC) was proposed, which uses information embedded in DCT coefficients during the encoding process. H.265 has introduced parallel processing features, which can speed-up this embedding process at the encoder side by using processors with a parallel processing capability. Unlike the MBs concept of H.264, a newly introduced quad-tree structure is adopted in H.265. Large Coding Units (LCUs) and Prediction Units (PUs) from the quad-tree structure of related regions are embedded to help reconstruction during concealment procedures. The embedding process is multi-level. This structure requires categorization of information based on its importance. The information of those most relevant regions is stored at the first level, while the least significant information is either stored at the last level or is discarded so as to reduce the storage requirement. There can be a situation where either the embedded information is lost or no information has been embedded. In such a situation, the information about the required blocks is extracted before the de-quantization process at the decoder side. The DHEC technique can recover both the Intra and Inter-encoded information. This proposed technique attempts to recover the lost information at its best and uses a simple procedure to select and embed the related information. 
The drawbacks of this approach are its increased bit rate and its unsuitability for consecutive data-loss situation.

\section{B. Motion-Compensated Error Concealment Based on Block Merging}

In [34], Motion Compensation-based Error Concealment (MCEC) was proposed, which utilizes the residual energy of MVs from a related Coding Unit (CU). One of the positive points of H.265 is that the information about CUs and PUs is kept safe during the encoding process. The proposed approach utilizes this saved information about CUs and PUs. The proposed technique uses two major assumptions. The first assumption is linear motion, which means that a lost or corrupted CU will follow the foot prints of its co-located CUs. The second assumption is that a lost or corrupted CU has the same partition structure as its nearby CUs. These assumptions lead to the usage of MVs of co-located CUs. To find out whether the selected MVs are reliable or not, the residual energy $(\mathrm{E})$ of the selected CU is calculated as the absolute sum of luma values by dividing the CU into PUs of size $4 \times 4$. If $E$ is smaller than a certain threshold, the selected PU is reliable and its MVs can be used; otherwise it is unreliable. The unreliable PUs are merged together and their corresponding MVs are replaced by a single MV. This single MV is the average of the MVs of those reliable PUs in the same CU. This algorithm maintains the useful information about object structure and produces a better reconstruction performance. It relies totally on nearby CUs, which makes it very vulnerable to consecutive data loss.

\section{Error Concealment for Whole-Frame Loss}

There is a possibility that a given frame of a video sequence can be encoded as a single packet or multiple packets, usually known as slices. Encoding a frame as a single slice makes it very vulnerable to bit errors. If even a single bit error occurs, the decoder will drop the entire frame. In [35], Motion Vector Extrapolation (MVE) [36] combined with variable block-size coding was proposed to recover the loss of a whole frame. In the proposed approach, the source of information is LCUs and PUs. Those regions of a frame with more motion are encoded with a smaller block size, while the remaining regions of the same frame are encoded with a larger block size. To detect the sizes of the different blocks in a lost frame, the block sizes and their corresponding MVs are analyzed during the concealment process. This decision is based on the temporal correlation (R) among the MVs of different blocks in the same frame. If the value of $\mathrm{R}$ is higher than a threshold, the corresponding blocks need to be subdivided; otherwise a larger block size is used. After detecting a suitable block size, texture and motion information about the reference frame is analyzed to predict the lost data. This method is computationally simple process and provides satisfactory results. One of the limitations of this approach is that it is suitable for linear motion only. Furthermore, it may produce blocky artifacts, and the estimated MVs may be inconsistent and not smooth.

\section{Error Concealment based on Block Partitioning}

In [37], block-partition decisions-based EC was proposed. In this approach, block-partitioning information is used in combination with MVE to segment the different objects in a reference frame. The block-partitioning information is obtained from the CUs and PUs of the referenced frame. This object segmentation helps in gathering MVs related to the same objects. After gathering related MVs and object segmentation information, those missing MVs and their corresponding objects can be estimated in the lost frame. Large-sized objects are given preference over smaller ones due to their consistent motion in nearby blocks. This approach is very similar to the approach presented in [35]. The only difference between these approaches is that the approach in [37] considers object segmentation for EC purpose. Because of their similarity, both approaches share the same advantages and disadvantages.

\section{CONCLUSION AND FUTURE RESEARCH DIRECTIONS}

In this paper, firstly, the need for EC techniques has been discussed. Secondly, three categories of EC techniques, i.e. Intra-frame EC, Inter-frame EC, and Hybrid EC, have been discussed. After that, the newly proposed EC schemes for H.265 were principally analyzed. For those techniques described in Section III, we have focused only on their visual qualities in terms of PSNR, and ignored their computational complexities. A possible reason for ignoring the computational complexity can be due to the fact that the HEVC standard is already very complex, and research to reduce its complexity is still ongoing. All of the newly proposed techniques are applied to HEVC to form a test-bed. Reducing the computational complexity of HEVC is another domain of research. According to the current research and the existing problems of the EC schemes, more attention should be paid to the following research issues:

- Intra frame-based EC techniques are usually not suitable for inter-frames. However, their importance cannot be ignored, as they help in estimating the structure of complex shapes, objects and sudden motion changes, and still need to be explored in H.265.

- The Intra frame-based EC methods rely on nearby MBs information. Such techniques face lots of difficulties when there is a continuous data loss. This research needs to be carried on, in order to deal with situations having continuous data loss or of less information by virtually utilizing MVs from previous frames.

- If Hybrid methods used for EC depend on the features of the referenced and surrounding MBs. If consecutive frames loss occurs, these methods will produce poor performance and will become difficult to detect required information.

- The object-based EC methods should attract more research effort because in real-time video processing, e.g., video conferencing, moving objects, are more important and should be visually clear.

- H.265 demands a higher computational complexity and is not suitable for devices with low-processing power. The 
complexity issue becomes more critical for consecutive frame loss in Inter-encoded video sequences.

\section{REFERENCES}

[1] Z. Cui, Z. Gan, X. Zhan and X. Zhu, "Error Concealment Techniques for Video Transmission over Error-Prone Channels: A Survey", Journal of Computational Information Systems, Vol. 28, No. 21, pp. 8807-8818, November 2012

[2] Y. Wang and Q. Zhu, "Error Control and Concealment for Video Communication: A Review”, Proceedings of IEEE, Vol. 80, No. 05, pp: 974-997, May 1998.

[3] B. Girod and N. Farber, "Feedback-Based Error Control for Mobile Video Transmission", Proceedings of IEEE, Vol. 87, No. 10, pp: 17071723, October 1999.

[4] Y. Zhang, C. Zhu and K. Yap, "A Joint Source-Channel Video Coding Scheme Based on Distributed Source Coding", IEEE Transactions on Multimedia, Vol. 10, No. 08, December 2008.

[5] J. Zhang and T. Tan, "Brief Review of Invariant Texture Analysis Methods", The Journal of Pattern Recognition Society, Vol. 35, No. 03, pp: 735-747, March 2002.

[6] W. Kumwilaisak and C. Kuo, "Spatial Error Concealment with Sequence-Aligned Texture Modeling and Adaptive Directional Recovery", Journal of Visual Communication and Image Representation, Vol. 22, pp: 164-177, December 2010.

[7] J. Koloda, J. Ostergaard and S. Jensen, "Sequential Error Concealment for Video/Images by Weighted Template Matching", Data Compression Conference, April 2012.

[8] J. Koloda, A. Peinado and V. Sanchez, "Kernel-Based MMSE Multimedia Signal Reconstruction and Its Application to Spatial Error Concealment", IEEE Transactions on Multimedia, Vol. 16, No. 06, October 2014

[9] http://en.wikipedia.org/wiki/Edge_detection

[10] H. Asheri, H. Rabiee, N. Pourdamghani and M. Ghanbari, "MultiDirectional Spatial Error Concealment using Adaptive Edge Thresholding", IEEE Transactions on Consumer Electronics, Vol. 58, No. 03, August 2012.

[11] M. Hoque, M. Pimentel, M. Hasan, K. Ahn and J. Kim, "Edge-Based Spatial Concealment of Digital Dropout Error in Degraded Archived Media", IET Electronics Letters, Vol. 50, No. 14, pp: 996-997, July 2014.

[12] J. Koloda, V. Sanchez and A. Peinado, "Spatial Error Concealment Based on Edge Visual Clearness for Image/Video Communication", Circuits, Systems and Signal Processing, October 2013.

[13] L. Zhu, Y. Zhao, S. Wang and H. Chen, "Spatial Error Concealment for Stereoscopic Video Coding Based on Pixel Matching", The Journal of Supercomputing, Vol. 58, No. 01, pp: 96-105, October 2011.

[14] V. Chellappa, P. Cosman and G. Voelker, "Error Concealment for Dual Frame Video Coding with Uneven Quality", Proceedings of Data Compression Conference, March 2005.

[15] http://www.wisegeek.com/what-is-the-difference-between-3d-and2d.htm

[16] S. Yang, C. Chang and C. Chan, "An Object Based Error Concealment Technique for H.264 Coded Video", Multimedia Tools and Applications, July 2014.

[17] W. Lie, C. Lee, C. Yeh and Z. Gao, "Motion Vector Recovery for Video Error Concealment by Using Iterative Dynamic Programming Optimization", IEEE Transactions on Multimedia, Vol. 16, No. 01, January 2014.

[18] X. Liu, W. Yang and Z. Shen, "H.264/AVC Video Error Concealment Algoritm by Employing Motion Vector Recovery Under Cloud Computing Environment", The Journal of Supercomputing, Vol. 70, No. 03, pp: 1180-1199, May 2014.

[19] Y. Zhang, X. Xiang, D. Zhao and W. Gao, "Packet Video Error Concealment with Auto Regressive Model", IEEE Transactions on
Circuits and Systems for Video Technology, Vol. 22, No. 01, January 2012.

[20] S. Cui, H. Cui and K. Tang, "An Effective Error Concealment Scheme for Heavily Corrupted H.264/AVC Videos Based on Kalman Filtering", Signal, Image and Video Processing, Vol. 08, No. 08, pp: 1533-1542, October 2012.

[21] H. Li and Y. Zhong, "Motion Characteristic Differentiated Error Concealment", Multimedia Tools and Applications, Vol. 65, No. 02, pp: 297-320, July 2013.

[22] Y. Zhao, C. Zhu, L. Yu and M. Tanimoto, "An Overview of 3D-TV System Using Depth-Image-Based Rendering”, 3D-TV System With Depth-Image-Based Rendering, Signals and Communication, Springer, 2013.

[23] B. Yan and J. Zhou, "Efficient Frame Concealment for Depth ImageBased 3-D Video Transmission", IEEE Transactions on Multimedia, Vol. 14, No. 03, June 2012.

[24] M. Yang, X. Lan, N. Zhang and P. Cosman, "Depth-Assisted Temporal Error Concealment for Intra Frame Slices in 3-D Video", IEEE Transactions on Broadcasting, Vol. 60, No. 02, June 2014.

[25] P. Lee, K. Kuo and C. Chi, "An Adaptive Error Concealment Method Based on Fuzzy Reasoning for Multi-View Video Coding”, Journal of Display Technology, Vol. 10, No. 07, July 2014.

[26] Y. Zhou, W. Xiang and G. Wang, "Frame Loss Concealment for MultiView Video Transmission Over Wireless Multimedia Sensor Networks", IEEE Sensors Journal, Vol. 15, No. 03, March 2015.

[27] L. Superiori, O. Nemethova and M. Rupp, "Error Concealment Analysis for H.264/Advanced Video Coding Encoded Video Sequences", E and I Electrical and Computer Engineering, Vol. 129, No. 06, pp: 387-399, October 2012.

[28] W.Tsai and J. Chen, "Joint Temporal and Spatial Error Concealment for Multiple Descripttion Video Coding", IEEE Transactions on Circuits and Systems for Video Technology, Vol. 20, No. 12, December 2010.

[29] J. Seiler, M. Schoberl and A.Kaup, "Spatio-Temporal Error Concealment in Video by Denoised Temporal Extrapolation Refinement", $20^{\text {th }}$ IEEE International Conference on Image Processing, pp: 1613-1616, September 2013.

[30] H. Asheri, H. Rabiee and M. Rohban, "Signal Extrapolation for Image and Video Error Concealment Using Gaussian Processes with Adaptive Nonstationary Kernels", IEEE Signal Processing Letters, Vol. 19, No. 10 , October 2012.

[31] J. Zhu and R. Dansereau, "Error-Resilient and Error Concealment 3-D SPIHT for Multiple Description Video Coding with Added Redundancy", IEEE Transactions on Circuits and Systems for Video Technology, Vol. 22, No. 06, June 2012.

[32] H. Hadizadeh, I. Bajic and G. Cheung, "Video Error Concealment Using a Computation Efficient Low Saliency Prior", IEEE Transactions on Multimedia, Vol. 15, No. 08, December 2013.

[33] F. Ramos, C. Uribe and R. Cumplido, "Video Error Concealment Based on Data Hiding for the Emerging Video Technologies", Lecture Notes in Computer Science, Image and Video Technology, 2014.

[34] Y. Chang and Z. Chen, "Motion Compensated Error Concealment for HEVC Based on Block Merging and Residual Energy", $20^{\text {th }}$ IEEE International Packet Video Workshop, December 2013.

[35] C. Liu, R. Ma and Z. Zhang, "Error Concealment for Whole Frame Loss in HEVC", Communications in Computer and Information Sciences, Advances on Digital Television and Wireless Multimedia Communications, Vol. 331, pp: 271-277, 2012.

[36] Q. Peng, T. Yang and C. Zhu, "Block-Based Temporal Error Concealment for Video Packet Using Motion Vector Extrapolation", IEEE International Conference on Communications, Circuits and Systems and West Sino Expositions, Vol. 01, pp: 10-14, July 2002.

[37] T. Lin, N. Yang and R. Syu, "Error Concealment Algorithm for HEVC Coded Video Using Block Partition Decisions", IEEE International Conference on Signal Processing, Communication and Computing (ICSPCC), August 2013. 